\title{
Very high energy gamma rays from the composite SNR G 0.9+0.1
}

\author{
F. Aharonian ${ }^{1}$, A. G. Akhperjanian ${ }^{2}$, K.-M. Aye ${ }^{3}$, A. R. Bazer-Bachi ${ }^{4}$, M. Beilicke ${ }^{5}$, W. Benbow ${ }^{1}$,
} D. Berge ${ }^{1}$, P. Berghaus ${ }^{6, \star}$, K. Bernlöhr ${ }^{1,7}$, C. Boisson ${ }^{8}$, O. Bolz $^{1}$, C. Borgmeier ${ }^{7}$, I. Braun ${ }^{1}$, F. Breitling ${ }^{7}$, A. M. Brown ${ }^{3}$, J. Bussons Gordo ${ }^{9}$, P. M. Chadwick ${ }^{3}$, L.-M. Chounet ${ }^{10}$, R. Cornils ${ }^{5}$, L. Costamante ${ }^{1,20}$,

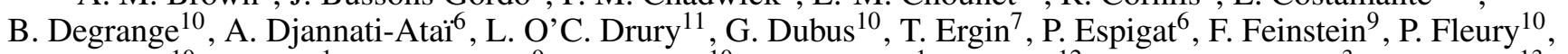
G. Fontaine ${ }^{10}$, S. Funk ${ }^{1}$, Y. A. Gallant ${ }^{9}$, B. Giebels ${ }^{10}$, S. Gillessen ${ }^{1}$, P. Goret $^{12}$, C. Hadjichristidis ${ }^{3}$, M. Hauser ${ }^{13}$, G. Heinzelmann ${ }^{5}$, G. Henri ${ }^{14}$, G. Hermann ${ }^{1}$, J. A. Hinton ${ }^{1}$, W. Hofmann ${ }^{1}$, M. Holleran ${ }^{15}$, D. Horns ${ }^{1}$, O. C. de Jager ${ }^{15}$, I. Jung ${ }^{1,13, \star \star}$, B. Khélifi ${ }^{1}$, Nu. Komin ${ }^{7}$, A. Konopelko ${ }^{1,7}$, I. J. Latham ${ }^{3}$, R. Le Gallou ${ }^{3}$, A. Lemière ${ }^{6}$, M. Lemoine ${ }^{10}$, N. Leroy ${ }^{10}$, T. Lohse ${ }^{7}$, A. Marcowith ${ }^{4}$, C. Masterson 1,20 , T. J. L. McComb ${ }^{3}$, M. de Naurois ${ }^{16}$, S. J. Nolan ${ }^{3}$, A. Noutsos ${ }^{3}$, K. J. Orford ${ }^{3}$, J. L. Osborne ${ }^{3}$, M. Ouchrif ${ }^{16,20}$, M. Panter ${ }^{1}$, G. Pelletier ${ }^{14}$, S. Pita ${ }^{6}$, G. Pühlhofer ${ }^{1,13}$, M. Punch ${ }^{6}$, B. C. Raubenheimer ${ }^{15}$, M. Raue ${ }^{5}$, J. Raux ${ }^{16}$, S. M. Rayner ${ }^{3}$, I. Redondo ${ }^{10,20, \star \star \star}$, A. Reimer ${ }^{17}$, O. Reimer ${ }^{17}$, J. Ripken ${ }^{5}$, L. Rob ${ }^{18}$, L. Rolland ${ }^{16}$, G. Rowell ${ }^{1}$, V. Sahakian ${ }^{2}$, L. Saugé ${ }^{14}$, S. Schlenker ${ }^{7}$, R. Schlickeiser ${ }^{17}$, C. Schuster ${ }^{17}$, U. Schwanke ${ }^{7}$, M. Siewert ${ }^{17}$, H. Sol ${ }^{8}$, R. Steenkamp ${ }^{19}$, C. Stegmann ${ }^{7}$, J.-P. Tavernet ${ }^{16}$, R. Terrier ${ }^{6}$, C. G. Théoret ${ }^{6}$, M. Tluczykont ${ }^{10,20}$, G. Vasileiadis ${ }^{9}$, C. Venter ${ }^{15}$, P. Vincent ${ }^{16}$, B. Visser ${ }^{15}$, H. J. Völk ${ }^{1}$, and S. J. Wagner ${ }^{13}$

\footnotetext{
1 Max-Planck-Institut für Kernphysik, PO Box 103980, 69029 Heidelberg, Germany e-mail: Jim.Hinton@mpi-hd.mpg.de

2 Yerevan Physics Institute, 2 Alikhanian Brothers St., 375036 Yerevan, Armenia

3 University of Durham, Department of Physics, South Road, Durham DH1 3LE, UK

4 Centre d'Étude Spatiale des Rayonnements, CNRS/UPS, 9 Av. du Colonel Roche, BP 4346, 31029 Toulouse Cedex 4, France

${ }^{5}$ Universität Hamburg, Institut für Experimentalphysik, Luruper Chaussee 149, 22761 Hamburg, Germany

${ }^{6}$ Physique Corpusculaire et Cosmologie, IN2P3/CNRS, Collège de France, 11 place Marcelin Berthelot, 75231 Paris Cedex 05, France

7 Institut für Physik, Humboldt-Universität zu Berlin, Newtonstr. 15, 12489 Berlin, Germany

${ }^{8}$ LUTH, UMR 8102 du CNRS, Observatoire de Paris, Section de Meudon, 92195 Meudon Cedex, France

9 Groupe d'Astroparticules de Montpellier, IN2P3/CNRS, Université Montpellier II, CC85, Place Eugène Bataillon, 34095 Montpellier Cedex 5, France

${ }^{10}$ Laboratoire Leprince-Ringuet, IN2P3/CNRS, École Polytechnique, 91128 Palaiseau, France

11 Dublin Institute for Advanced Studies, 5 Merrion Square, Dublin 2, Ireland

12 Service d'Astrophysique, DAPNIA/DSM/CEA, CE Saclay, 91191 Gif-sur-Yvette, France

13 Landessternwarte, Königstuhl, 69117 Heidelberg, Germany

14 Laboratoire d'Astrophysique de Grenoble, INSU/CNRS, Université Joseph Fourier, BP 53, 38041 Grenoble Cedex 9, France

15 Unit for Space Physics, North-West University, Potchefstroom 2520, South Africa

${ }^{16}$ Laboratoire de Physique Nucléaire et de Hautes Énergies, IN2P3/CNRS, Universités Paris VI \& VII, 4 place Jussieu, 75231 Paris Cedex 05, France

17 Institut für Theoretische Physik, Lehrstuhl IV: Weltraum und Astrophysik, Ruhr-Universität Bochum, 44780 Bochum, Germany

18 Institute of Particle and Nuclear Physics, Charles University, V Holesovickach 2, 18000 Prague 8, Czech Republic

19 University of Namibia, Private Bag 13301, Windhoek, Namibia

20 European Associated Laboratory for Gamma-Ray Astronomy, jointly supported by CNRS and MPG
}

Received 15 December 2004 / Accepted 11 January 2005

\footnotetext{
* Université Libre de Bruxelles, Faculté des Sciences, Campus de la Plaine, CP230, Boulevard du Triomphe, 1050 Bruxelles, Belgium.

$\star \star$ Now at Washington Univ., Department of Physics, 1 Brookings Dr., CB 1105, St. Louis, MO 63130, USA.

$\star \star \star$ Now at Department of Physics and Astronomy, Univ. of Sheffield, The Hicks Building, Hounsfield Road, Sheffield S3 7RH, UK.
} 
Abstract. Very high energy ( $>100 \mathrm{GeV}$ ) gamma-ray emission has been detected for the first time from the composite supernova remnant G $0.9+0.1$ using the HESS instrument. The source is detected with a significance of $\approx 13 \sigma$, and a photon flux above $200 \mathrm{GeV}$ of $\left(5.7 \pm 0.7_{\text {stat }} \pm\right.$ $\left.1.2_{\text {sys }}\right) \times 10^{-12} \mathrm{~cm}^{-2} \mathrm{~s}^{-1}$, making it one of the weakest sources ever detected at $\mathrm{TeV}$ energies. The photon spectrum is compatible with a power law $\left(\mathrm{d} N / \mathrm{d} E \propto E^{-\Gamma}\right)$ with photon index $\Gamma=2.40 \pm 0.11_{\text {stat }} \pm 0.20_{\text {sys }}$. The gamma-ray emission appears to originate in the plerionic core of the remnant, rather than the shell, and can be plausibly explained as inverse Compton scattering of relativistic electrons.

Key words. ISM: supernova remnants - ISM: individual objects: G 0.9+0.1 - gamma-rays: observations

\section{Introduction}

G0.9+0.1 is a well known composite supernova remnant, recognised as such from its radio morphology (Helfand \& Becker 1987). It exhibits a bright compact core $\left(\sim 2^{\prime}\right.$ across $)$ surrounded by an $8^{\prime}$ diameter shell. The radio spectrum of the core is significantly harder $(\alpha \approx 0.12)$ than that of the shell ( $\alpha \approx-0.77$ ) (LaRosa et al. 2000). X-ray observations of the nebula with BeppoSAX (Mereghetti et al. 1998), Chandra (Gaensler et al. 2001) and XMM-Newton (Porquet et al. 2003) have unambiguously identified the core region as a pulsar wind nebula $(\mathrm{PWN})$. A plausible candidate for the central pulsar is the hard spectrum point source CXOU J174722.8-280915; however no pulsed emission has been detected. Observations with XMM have revealed a softening of the X-ray spectrum with increasing distance from the centre of the PWN, a signature of energy loss of electrons within the nebula. The location of $\mathrm{G} 0.9+0.1$ in the Galactic Centre (GC) region suggests a distance of $\sim 8.5 \mathrm{kpc}$ (Mezger et al. 1996). At this distance the size of the shell implies an age of a few thousand years for the SNR. At gammaray energies $\mathrm{G} 0.9+0.1$ has not been detected previously. It is not associated with any EGRET source and the only published observation in the $\mathrm{TeV}$ domain is an unconstraining upper limit from the HEGRA collaboration (flux $<4.6 \times 10^{-12} \mathrm{~cm}^{-2} \mathrm{~s}^{-1}$ above 4.2 TeV, Aharonian et al. 2002).

The High Energy Stereoscopic System (HESS) is a newly completed instrument designed to study astrophysical gamma radiation in the energy range $100 \mathrm{GeV}-10 \mathrm{TeV}$. HESS consists of four imaging atmospheric Cherenkov telescopes (Hinton 2004; Bernlöhr et al. 2003; Vincent et al. 2003; Aharonian et al. 2004a); shower images are required in at least two of them to trigger the detector (Funk et al. 2004). Using the stereoscopic technique the instrument reaches an angular resolution of $\sim 0.1^{\circ}$ and a point source sensitivity of $1 \%$ of the flux from the Crab Nebula $(5 \sigma$ in $25 \mathrm{~h})$.

Observations of the GC region were made with the partially complete HESS system in 2003. These data revealed emission from the Sgr A region, consistent with the position of Sgr A* (Aharonian et al. 2004b). Within the same field of view indications for a signal from the supernova remnant $\mathrm{G} 0.9+0.1$ were seen at the $4 \sigma$ level. A relatively deep HESS observation of this region with the complete telescope system was performed in March-September 2004. The results from this dataset relevant to $\mathrm{G} 0.9+0.1$ are presented here. A detailed discussion of the implications of the 2004 dataset for the region around Sgr A* will be presented elsewhere.

\section{HESS observations and results}

The large field of view of the HESS cameras $\left(\sim 5^{\circ}\right)$ provides good sensitivity for point sources at an angular distance up to $\sim 2^{\circ}$ from the pointing direction of the telescope system. The bulk of observations presented here were taken in wobble mode around the position of $\mathrm{Sgr} \mathrm{A}^{*}$. In this mode 28-min runs are taken pointing $0.5^{\circ}$ away from the nominal source in alternating directions. Approximately $20 \%$ of the data were taken in wobble mode around G0.9+0.1 itself. The observations have an average offset of $0.9^{\circ}$ from the SNR. The offaxis sensitivity of the system derived from Monte Carlo simulations has been confirmed via observations of the Crab Nebula (Aharonian et al. 2004c). The total live time of the observations after run selection is $50 \mathrm{~h}$, from a total observation time of $60 \mathrm{~h}$. The mean zenith angle of observation was $18^{\circ}$, resulting in an energy threshold of $170 \mathrm{GeV}$ after standard cuts.

Standard event reconstruction was applied to these data: tail-cuts image cleaning, Hillas parameterisation and stereoscopic direction reconstruction based on the intersection of image axes (Aharonian et al. 2005). Cuts on the scaled width and length of images (optimised on gamma-ray simulations and offsource data) are made to suppress the hadronic background. An additional cut on the minimum size of images ( 200 photoelectrons) is made to further suppress the background and select a subset of events with a superior angular resolution $\left(0.07^{\circ} \mathrm{rms}\right)$, but with an increased energy threshold of $350 \mathrm{GeV}$. Events with directions reconstructed within an angle $\theta$ of a trial source location are considered on-source where $\theta<0.1^{\circ}$. A ring of radius $0.5^{\circ}$ and an area 7 times that of the on-source region is used to derive a background estimate. The on-source and background region counts, together with a normalisation factor for the different acceptance of these regions, are used to derive the statistical significance of any excess following the likelihood method of Li \& Ma (1983).

Figure 1 shows a significance sky map for the field of view of the HESS GC observations derived as described above. Two sources of very high energy (VHE) gamma-rays are clearly visible: the Galactic Centre (HESS J1745-290) and a second source (HESS J1747-281) coincident with the SNR G 0.9+0.1. The statistical significance of HESS J1745-290 is 35 $\sigma$ in this dataset, compared with $11 \sigma$ in our 2003 data (Aharonian et al. 2004b). This increase is expected (for a steady source) from the greater sensitivity of the full 4-telescope array with respect to the 2-telescope configuration used in 2003, and from the increased exposure time in 2004.

The HESS 2003 dataset showed evidence for gamma-ray emission at the position of G $0.9+0.1$ at the $4 \sigma$ level. This evidence is confirmed by the presence of a $13 \sigma$ excess at the 


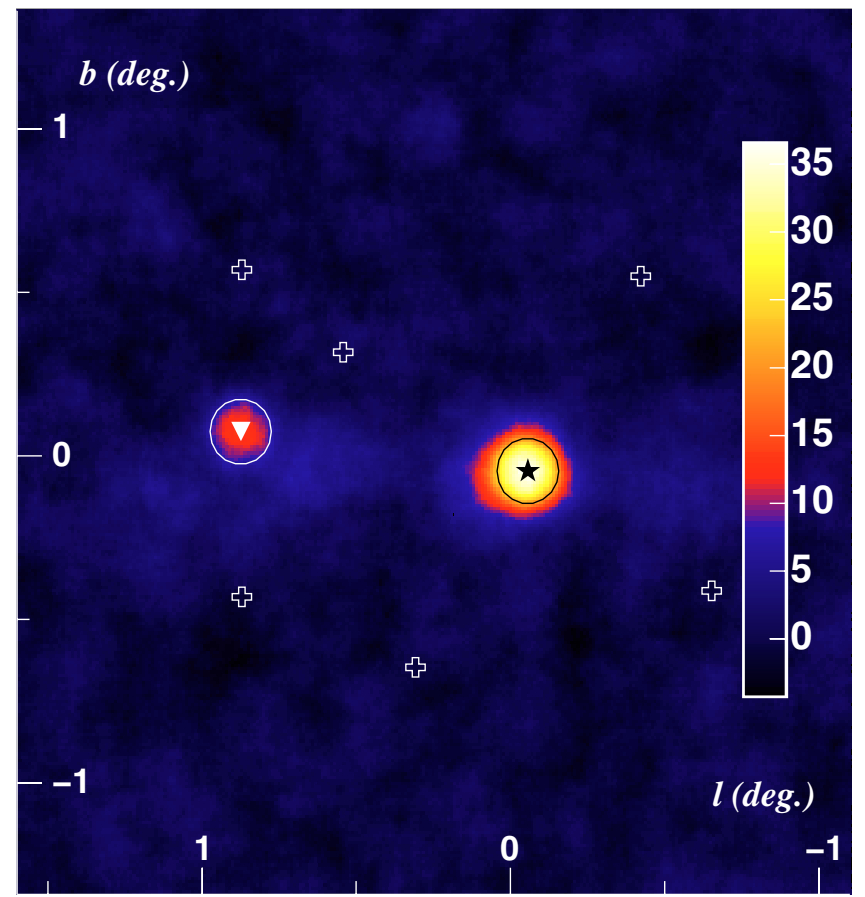

Fig. 1. Gamma-ray point source significance map for the region around the GC. The position of $\mathrm{G} 0.9+0.1$ is marked with a triangle. $\mathrm{Sgr} \mathrm{A}^{*}$ is marked with a star. The circles show the integration regions contributing to the quoted significance at the positions of these two objects. The six telescope pointings are shown as crosses.

same position in the independent 2004 dataset. The gammaray like excess is well fit by the point spread function of the instrument, yielding a best fit position of $l=0.872^{\circ} \pm 0.005^{\circ}$, $b=0.076^{\circ} \pm 0.005^{\circ}$, consistent within statistical errors with the position of the PWN in G0.9+0.1 $\left(l=0.871^{\circ}, b=\right.$ $\left.0.0772^{\circ}\right)($ Gaensler et al. 2001). The systematic pointing error is approximately $20^{\prime \prime}$ in each direction for these data.

The simulated point-spread function of the instrument provides an adequate description of the excess. Assuming a radially symmetric Gaussian emission region $(\rho \propto$ $\left.\exp \left(-\theta^{2} / 2 \sigma_{\text {source }}^{2}\right)\right)$ a $95 \%$ confidence limit on the extension $\sigma_{\text {source }}<1.3^{\prime}$ is obtained. For emission from a uniform thin shell a limit of $2.2^{\prime}$ on the shell radius can be derived at the same confidence level. Figure 2 shows a radio map of G0.9+0.1 at $90 \mathrm{~cm}$ (LaRosa et al. 2000) showing the SNR shell and the central compact source (colour scale). Contours of VHE gamma-ray emission are superimposed along with the point spread function of the instrument for comparison. The reconstructed gamma-ray source position is marked as a cross. Given the close positional coincidence, we identify the VHE gamma-ray source HESS J1747-281 with G 0.9+0.1.

Figure 3 shows the reconstructed gamma-ray spectrum of G 0.9+0.1 using two sets of event selection cuts. A size cut of 80 photoelectrons (pe) is applied to extend the spectrum to lower energies. This dataset can be fit by a power law in energy with photon index $2.40 \pm 0.11_{\text {stat }} \pm 0.20_{\text {sys }}$ and a flux above $200 \mathrm{GeV}$ of $\left(5.7 \pm 0.7_{\text {stat }} \pm 1.2_{\text {sys }}\right) \times 10^{-12} \mathrm{~cm}^{-2} \mathrm{~s}^{-1}$. The fit has a $\chi^{2} /$ d.o.f. of $3.5 / 5$. Using the harder image size cut of 200 pe used elsewhere in this paper yields consistent results (photon

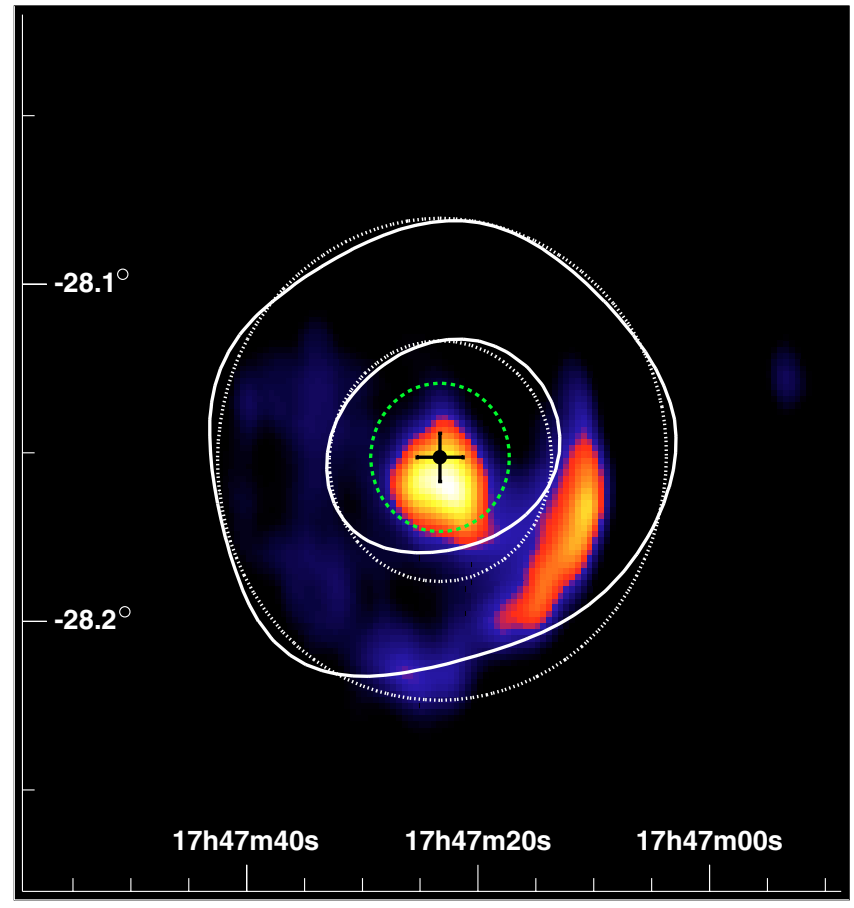

Fig. 2. $90 \mathrm{~cm}$ radio flux map of G 0.9+0.1 from LaRosa et al. (2000) (colour scale), overlayed with contours (at $40 \%$ and $80 \%$ peak brightness) of the smoothed and acceptance corrected count map of gammaray candidates (solid lines). The HESS data are smoothed with a Gaussian of rms $0.03^{\circ}$ to reduce statistical fluctuations. The simulated point-spread function of the instrument is shown with dotted lines (also $40 \%$ and $80 \%$ ). The best fit position of the VHE gamma-ray excess is shown with error bars showing combined statistical and systematic errors. The innermost circle (dashed) illustrates the 95\% confidence limit on the rms size of the emission region.

index $2.29 \pm 0.14_{\text {stat }}$ and a flux of $\left(5.5 \pm 0.8_{\text {stat }}\right) \times 10^{-12} \mathrm{~cm}^{-2} \mathrm{~s}^{-1}$ above $200 \mathrm{GeV}$ ). This flux represents only $2 \%$ of the flux from the Crab Nebula above the $200 \mathrm{GeV}$ spectral analysis threshold. Although G $0.9+0.1$ is one of the weakest sources ever detected at $\mathrm{TeV}$ energies, the detection presented here is statistically highly significant $(13 \sigma)$, demonstrating the sensitivity of the HESS instrument.

\section{Discussion}

$\mathrm{X}$-ray emission from the shell of G $0.9+0.1$ is only marginally detected above the local GC diffuse emission with a flux of $2 \times 10^{-12} \mathrm{erg} \mathrm{cm}^{-2} \mathrm{~s}^{-1}$ in the range $2-10 \mathrm{keV}$, and is consistent with both a thermal or non-thermal origin (Porquet et al. 2003). The non-thermal flux from the PWN is $5.8 \times 10^{-12} \mathrm{erg} \mathrm{cm}^{-2} \mathrm{~s}^{-1}$ in the same energy range, corresponding to a luminosity of $\sim 5 \times$ $10^{34} \mathrm{erg} \mathrm{s}^{-1}$ assuming a distance of $8.5 \mathrm{kpc}$. The lack of strong non-thermal X-ray emission from the shell and the point-like nature of the HESS detection argue against an origin of the VHE emission in the shell. Thus the PWN appears to be a more compelling source for the VHE gamma-rays.

The total power radiated by $\mathrm{G} 0.9+0.1$ in VHE gamma-rays is $\sim 2 \times 10^{34} \mathrm{erg} \mathrm{s}^{-1}$ (at $8.5 \mathrm{kpc}$ ), compared with $\sim 4 \times 10^{34} \mathrm{erg} \mathrm{s}^{-1}$ for the Crab Nebula (at $2 \mathrm{kpc}$ ) in the energy band $0.2-10 \mathrm{TeV}$. 


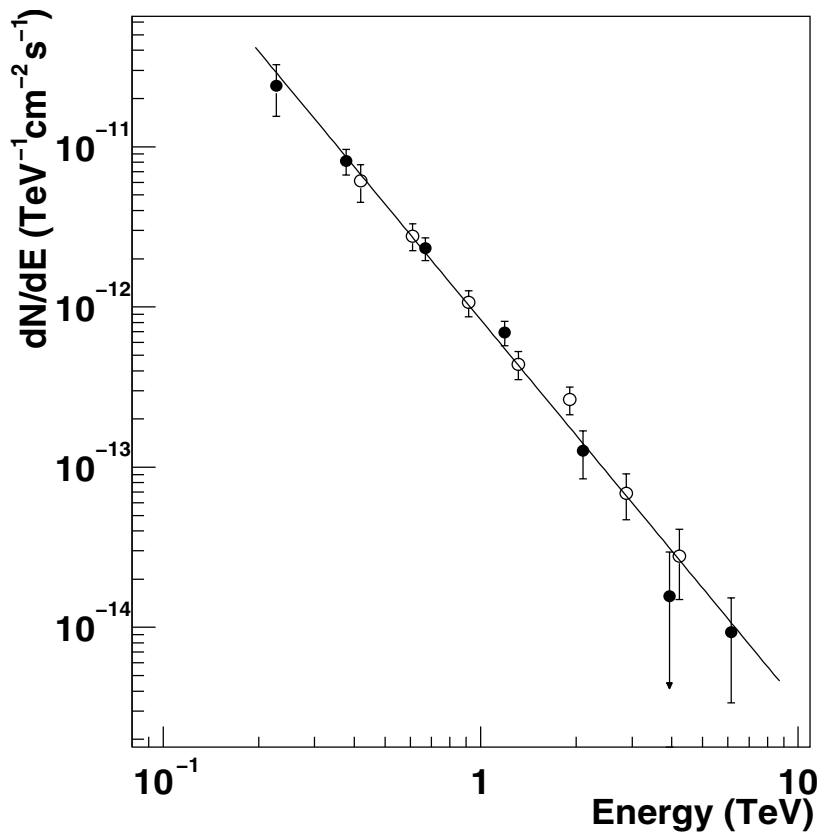

Fig. 3. Reconstructed VHE gamma-ray spectrum of G 0.9+0.1. Empty circles show the spectrum derived using the rather tight cuts used for Figs. 1 and 2. Solid symbols show the spectrum derived using an image size cut of 80 photoelectrons (optimised for maximal significance for $10 \%$ Crab strength sources) rather than 200 photoelectrons. The line shows a power-law fit to the 80 pe data set.

Until now the Crab Nebula represented the only firm identification of VHE emission from a nebula powered by a young neutron star.

A simple one-zone inverse Compton model for the VHE gamma-ray emission (Khélifi 2002) can be used to explain the observed data. A parent population of accelerated electrons with a broken power-law spectrum is assumed. The magnetic field strength $B$ is assumed to be uniform within the PWN and is a free parameter. The maximum energy of the electron spectrum is kept fixed at $500 \mathrm{TeV}$ as it can not be derived due to the absence of measurements in the hard $\mathrm{X}$-ray/soft $\gamma$-ray band. The synchrotron and inverse Compton (IC) emission of these electrons are calculated according to Blumenthal \& Gould (1970). The Klein-Nishina effect on the IC cross section is taken into account.

For sources in the GC region the presence of radiation densities considerably higher than typical Galactic Plane values leads to substantially enhanced IC emission (de Jager et al. 1995). The seed photons for IC scattering are the cosmic microwave background radiation (CMBR), the galactic diffuse emission around $100 \mu \mathrm{m}$ from dust and around $1 \mu \mathrm{m}$ from starlight. The energy spectra of the dust and starlight components are taken from a recent estimation of the interstellar radiation field (ISRF) used for the GALPROP code (Strong et al. 2000). To account for possible local variations of the starlight component the energy density of this component is kept as a free parameter. The energy density of the dust ISRF is kept fixed at $0.23 \mathrm{eV} \mathrm{cm}^{-3}$ as used in GALPROP. The starlight ISRF energy density, the electron spectrum parameters and $B$

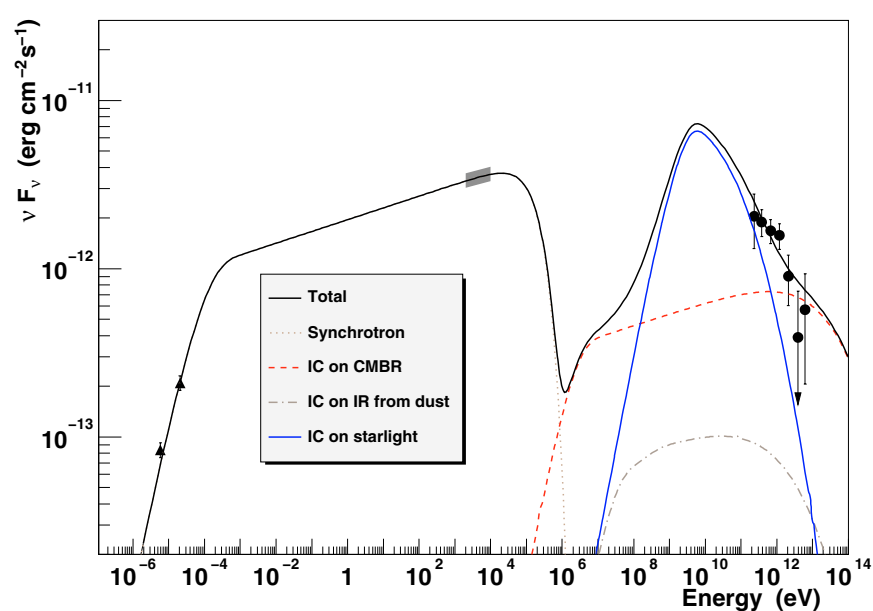

Fig. 4. The spectral energy distribution of the PWN in G0.9+0.1 from radio to VHE gamma-ray. Radio data (triangles) are taken from (Helfand \& Becker 1987), X-ray data (shaded box) from Porquet et al. 2003. The circles show the HESS data from this work. The solid curve shows a fit of a one-zone inverse Compton model to all data. Contributions of the CMBR, IR from dust and starlight photon fields to the IC emission are shown.

are adjusted such that the computed synchrotron and IC spectra match the observations.

Figure 4 shows the broad-band spectral energy distribution (SED) of G $0.9+0.1$, together with the best model fit. The fitted mean magnetic field strength is $\approx 6 \mu \mathrm{G}$. The model electron spectrum has a low energy index of 0.6 and a high energy index of 2.9 with a break at $25 \mathrm{GeV}$. The best fit energy density of the starlight ISRF is about $5.7 \mathrm{eV} \mathrm{cm}^{-3}$, that is $50 \%$ smaller than the value used in GALPROP. This derived value of $B$ is very close to the equipartition magnetic field $(\approx 5 \mu \mathrm{G})$, calculated from the whole fitted electron spectrum and given a source of radius $1^{\prime}$ at $8.5 \mathrm{kpc}$. Given the observed variation of the X-ray spectrum within the PWN, this one zone model is clearly somewhat simplistic. However, such a model, describing the average properties of the PWN, can describe the available data with reasonable physical parameters. Future measurements in the $\mathrm{GeV}$ regime with GLAST (sensitivity $2 \times 10^{-12} \mathrm{erg} \mathrm{s}^{-1} \mathrm{~cm}^{-2}$ at $1 \mathrm{GeV}$ ) are needed to resolve the ambiguity between the magnetic energy density and that of target radiation fields in this region. Alternative explanations based on a hadronic origin of the VHE emission (for example via the decay of neutral pions produced in $p-p$ collisions) are not excluded.

The detection of VHE gamma-ray emission from $\mathrm{G} 0.9+0.1$ provides the first direct evidence for the acceleration of very energetic particles in this object. The position and point-like nature of the gamma-ray emission, combined with the broad band SED of G $0.9+0.1$, strongly suggest an origin of the VHE emission in the compact central source (previously identified as a pulsar wind nebula).

This detection represents the first step towards the study of an emerging population of VHE gamma-ray emitting PWN. The detection of new PWN with accurate TeV spectra and with morphological measurements will provide information key to understanding particle acceleration in the vicinity of pulsars. 
Acknowledgements. The support of the Namibian authorities and of the University of Namibia in facilitating the construction and operation of HESS is gratefully acknowledged, as is the support by the German Ministry for Education and Research (BMBF), the Max Planck Society, the French Ministry for Research, the CNRS-IN2P3 and the Astroparticle Interdisciplinary Programme of the CNRS, the UK Particle Physics and Astronomy Research Council (PPARC), the IPNP of the Charles University, the South African Department of Science and Technology and National Research Foundation, and by the University of Namibia. We appreciate the excellent work of the technical support staff in Berlin, Durham, Hamburg, Heidelberg, Palaiseau, Paris, Saclay, and in Namibia in the construction and operation of the equipment. We would also like to thank J. Lazio (NRL) for provision of $90 \mathrm{~cm}$ radio data and A. W. Strong for providing a parameterisation of interstellar radiation fields.

\section{References}

Aharonian, F., Akhperjanian, A. G., \& Beilicke, M. 2002, A\&A, 395, 803

Aharonian, F., Akhperjanian, A. G., Aye, K.-M., et al. 2004a, Astropart. Phys., 22, 109

Aharonian, F., Akhperjanian, A. G., Aye, K.-M., et al. 2004b, A\&A, 425, L13

Aharonian, F., Akhperjanian, A. G., Aye, K.-M., et al. 2004c, in preparation
Aharonian, F., Akhperjanian, A. G., Aye, K.-M., et al. 2005, A\&A, 430,865

Bernlöhr, K., Carrol, O., Cornils, R., et al. 2003, APh, 20, 111

Blumenthal, G. R., \& Gould, R. J. 1970, RvMP, 42 (2), 237

Funk, S., Hermann, G., Hinton, J., et al. 2004, Astropart. Phys., 22, 285

Gaensler, B. M., Pivovaroff, M. J., \& Garmire, G. P. 2001, ApJ, 556, L107

Helfand, D. J., \& Becker, R. H. 1987, ApJ, 314, 203

Hinton, J. A. (HESS Collaboration) 2004, New Astron. Rev., 48, 331

de Jager, O. C., Harding, A. K., Baring, M. G., \& Mastichiadis, A. 1995, Proc. 24th ICRC (Rome), 528

Khélifi, B. 2002, Ph.D. Thesis, University of Caen, France (http://lpnp90.in2p3.fr/ cat/Thesis/)

LaRosa, T. N., Kassim, N. E., Lazio, T. J. W., \& Hyman, S. D. 2000 , AJ, 119, 207

Li, T., \& Ma, Y. 1983, ApJ, 272, 317

Mereghetti, S., Sidoli, L., \& Israel, G. L. 1998, A\&A, 331, L77

Mezger, P. G., Duschl, W. J., \& Zylka, R. 1996, A\&ARv, 7, 289

Porquet, D., Decourchelle, A., \& Warwick, R. S. 2003, A\&A, 401, 197

Strong, A. W., Moskalenko, I. V., \& Reimer, O. 2000, ApJ, 537, 763

Vincent, P., Denance, J.-P., Huppert, J.-F., et al. 2003, Proc. 28th ICRC, Tsukuba (Tokyo: Univ. Academy Press), 2887 